\title{
Naissance et renaissance du conte de fées : de Marie-Catherine d'Aulnoy à Angela Carter
}

\section{Magali Monnier}

\section{(2) OpenEdition}

\section{Journals}

Édition électronique

URL : http://journals.openedition.org/edl/206

DOI : $10.4000 /$ edl.206

ISSN : 2296-5084

Éditeur

Université de Lausanne

\section{Édition imprimée}

Date de publication : 15 décembre 2011

Pagination : 243-258

ISBN : 978-2-940331-26-0

ISSN : 0014-2026

\section{Référence électronique}

Magali Monnier, « Naissance et renaissance du conte de fées : de Marie-Catherine d'Aulnoy à Angela Carter », Études de lettres [En ligne], 3-4 | 2011, mis en ligne le 15 décembre 2014, consulté le 18

décembre 2020. URL : http://journals.openedition.org/edl/206 ; DOI : https://doi.org/10.4000/edl.206 


\section{NAISSANCE ET RENAISSANCE DU CONTE DE FÉES: DE MARIE-CATHERINE D'AULNOY À ANGELA CARTER}

Cet article propose une lecture croisée de la dimension métapoétique dans "Gracieuse et Percinet» et "La Chatte blanche» de Marie-Catherine d'Aulnoy, auteure française du XVII ${ }^{\mathrm{e}}$ siècle, et dans "The Courtship of Mr Lyon» d'Angela Carter, une réécriture moderne "féministe» de "La Belle et la Bête» qui rend aussi hommage à d'Aulnoy. L'analyse comparative montre que ces textes sont liés par un dialogue intertextuel qui porte sur des éléments à la fois thématiques et esthétiques, et qu'ils partagent une même préoccupation pour le genre littéraire en relation avec la politique de genre (gender politics). Ainsi, ces textes représentent le genre du conte de fées comme un espace féminin privilégié, que d'Aulnoy inaugure et dont Carter assure à la fois la continuité et le renouvellement au $\mathrm{XX}^{\mathrm{e}}$ siècle en filant la métaphore architecturale du Palais de Féerie chère à d'Aulnoy.

\section{Marie-Catherine d'Aulnoy et Angela Carter, les fées marraines du conte de fées littéraire}

Les Parques et les fées partagent un lien étroit avec le destin et la parole. Dans son étude du mythe littéraire des Parques, Sylvie Ballestra-Puech rappelle que les déesses qui filent le destin des mortels sont parfois appelées Fata ${ }^{1}$. Ce nom dérive probablement de fatum, le destin, qui provient lui-même de fari, "parler», et «met en lumière une composante fondamentale du mythe, qui nous semble proprement latin: l'accent mis sur

Je remercie vivement Martine Hennard Dutheil de la Rochère pour son soutien et son aide précieuse dans l'élaboration de ma réflexion sur le Palais de Féerie et la rédaction de cet article.

I. S. Ballestra-Puech, Les Parques, p. 40-45. 
la parole prophétique». Or, fata constitue la racine étymologique du mot "fée». La figure de la fée marraine dont les paroles déterminent le sort des nouveau-nés apparaît bien comme l'héritière des Fata antiques ${ }^{2}$. Ainsi, il suffit aux fées qui se penchent sur le berceau de la Belle au Bois Dormant de prononcer des vœux pour que ceux-ci se réalisent en dons qui décident de l'avenir de la jeune princesse. Ce pouvoir n'est pas sans évoquer celui des écrivains sur leurs personnages, dont ils guident les pas à travers le récit. Cet article s'appuie sur cette similitude pour analyser la relation entre fée, parole et destin dans son rapport avec le genre du conte de fées littéraire tel qu'il est instauré par Marie-Catherine d'Aulnoy, à qui on doit d'ailleurs le terme générique de "conte de fées" ou "conte des fées", dont l'ambiguïté est significative; à son tour, Angela Carter revisitera et renouvellera le genre du conte (ou plutôt du fairy tale) au $\mathrm{XX}^{\mathrm{e}}$ siècle.

Marie-Catherine d'Aulnoy est la première à utiliser le terme "conte des fées" en France, dans le recueil du même nom paru en 1697, la même année que les Histoires ou Contes du temps passé, avec des Moralités de Perrault. Elle faisait partie d'un cénacle de conteuses qui s'assimilaient aux fées et utilisaient parfois ce terme pour se désigner mutuellement. Les fées des contes de Marie-Catherine d'Aulnoy sont d'ailleurs souvent des figures de conteuses ${ }^{3}$. Sylvie Ballestra-Puech montre par exemple comment l'auteure fait de "Serpentin vert» un véritable manifeste esthétique en condensant dans le personnage de Laidronnette et l'épreuve de filage qui lui est imposée les figures de la Parque, de la fée et d'Arachné ${ }^{4}$. L'auteure élabore donc dans ses contes une réflexion approfondie sur la poétique du genre en constitution qu'est le conte littéraire, sa propre pratique de conteuse et le rôle social que ce statut lui confère. Elle participe ainsi aux débats littéraires, esthétiques et sociaux de son époque, car le

2. Pour une analyse détaillée de cette filiation, voir ibid., p 59-66 et 189-209, ainsi que les contributions de V. Dasen, S. Ballestra-Puech, D. Haase et M. Hennard Dutheil de la Rochère dans ce volume, qui abordent la dimension performative de la parole et ses implications métatextuelles.

3. Pour des analyses plus détaillées des représentations de la conteuse à travers la figure de la fée chez d'Aulnoy et ses consœurs, voir notamment G. Verdier, «Figures de la conteuse dans les contes de fées féminins»; L. Seifert, "Les Fées Modernes»; id., Fairy Tales, Sexuality and Gender in France 1690-1715 et R. Böhm, "La participation des fées modernes à la création d'une mémoire féminine».

4. Voir l'article de S. Ballestra-Puech dans ce volume. 
choix du conte de fées comme genre de prédilection la situe résolument dans la fameuse Querelle des Anciens et des Modernes en faveur de ces derniers.

Angela Carter est également très consciente de la vive controverse autour du conte qui agite les milieux féministes anglo-américains lorsqu'elle publie The Bloody Chamber and Other Stories en 1979. De nombreuses voix s'élèvent alors pour dénoncer les contes de «La Belle au bois dormant ", "Cendrillon» ou "Blanche Neige», accusés de perpétuer des stéréotypes, normes et valeurs patriarcales ${ }^{5}$. A travers ses réécritures du "Petit Chaperon Rouge», de "La Belle et la Bête» et de "Barbe Bleue", Carter prend une part active à ce débat et formule une position propre qui a valeur de manifeste esthétique et politique. Ses récits, qu'elle qualifie elle-même de "stories about fairy stories" ${ }^{6}$, mettent en lumière le fonctionnement des contes dont elle s'inspire et en proposent des interprétations potentiellement émancipatrices. Cette "relecture» critique et créative des contes lui permet de montrer que le genre est propice à l'expérimentation et à la réflexion, et se prête à la formulation d'une poétique féministe déjà amorcée dans l'œuvre de celles qui l'ont précédée. Carter renouvelle ainsi véritablement le genre du fairy tale de même que sa réception critique ${ }^{7}$.

D’Aulnoy et Carter partagent donc une même démarche métapoétique au sens où leurs textes mettent en scène et commentent implicitement leur propre structure, style, contenu thématique et discours sur le genre (dans le double sens de forme littéraire et rapports de sexe), ainsi que leur inscription dans un contexte social, culturel et littéraire spécifique. Elles font de cette réflexivité une caractéristique du genre du conte, sur le développement duquel toutes deux exercent une influence

5. Ces jugements sont fréquemment basés sur des éditions populaires des contes de Perrault ou des Grimm, ou sur les films de Disney qui s'en inspirent. L'analyse des contes écrits dans leur version originale révèle une réalité bien plus nuancée, comme le montre M. Hennard Dutheil de la Rochère dans " But Marriage Itself is no Party" ", p. 131-132).

6. A. Carter, "Notes from the Front Line», p. 38. Cette expression explicite de manière frappante la réflexivité des récits de Carter.

7. Voir D. Haase, «Feminist Fairy-Tale Scholarship», pour un compte-rendu détaillé de l'évolution des débats critiques autour du fairy tale. 
décisive à leur époque respective ${ }^{8}$. En cela, elles sont donc semblables à des Parques filant le destin du conte de fées littéraire, ou à des fées marraines définissant les qualités d'un genre qui s'élabore pour l'une, et qui se renouvelle et se réinvente pour l'autre 9 .

Cette hypothèse sera illustrée par une analyse comparative de trois textes qui marquent selon moi trois moments distincts de l'histoire du conte de fées littéraire. Le premier tome des Contes des fées s'ouvre sur "Gracieuse et Percinet». Je montrerai qu'il s'agit d'un conte programmatique, qui représente métaphoriquement l'entrée de l'auteure dans la féerie à partir de la métaphore architecturale du palais merveilleux. "La Chatte Blanche" fait partie des Contes nouveaux ou les fées à la mode, le second recueil de Marie-Catherine d'Aulnoy publié en 1698, que je propose de lire comme une mise en scène du triomphe du conte de fées et de la conteuse. "The Courtship of Mr Lyon", quant à lui, est issu de The Bloody Chamber and Other Stories, le désormais célèbre recueil de réécritures de contes d'Angela Carter. Celui-ci revisite certains contes considérés comme classiques à la fin du $\mathrm{XX}^{\mathrm{e}}$ siècle et met en évidence les possibilités de renouvellement du genre à partir d'une perspective féministe. Le lien entre ces textes est d'autant plus fort que "La Chatte Blanche» fait directement allusion à "Gracieuse et Percinet» et que "The Courtship of Mr Lyon» renvoie explicitement à «La Chatte Blanche». Il y a donc continuité, mais aussi démarche critique et innovation. Mon approche de ce corpus est à la fois textuelle et transtextuelle. Elle porte aussi bien sur les éléments qui font la cohérence interne de chaque texte, comme les motifs, le style ou la structure, que sur leur dimension intertextuelle à partir des principes de la comparaison différentielle proposés par Ute Heidmann ${ }^{10}$. L'analyse de ces textes se concentrera sur un motif

8. S. Benson parle de "Carter generation" pour qualifier les écrivains contemporains retravaillant à divers degrés le genre du fairy tale (S. Benson, "Introduction", p. 4). Il affirme que The Bloody Chamber "has had a profound and pervasive impact on our understanding of and engagement with the fairy tale» (ibid., p. 13).

9. N. Jasmin a déjà proposé cette analogie dans le cas de Marie-Catherine d'Aulnoy (N. Jasmin, Naissance du conte féminin, p. 381-389). E. W. Harries souligne également la réflexivité dans les textes des conteuses et postule que Carter se positionne dans la continuité de cette stratégie (E. W. Harries, Twice Upon a Time, p. 15, 17 et 32). Il n'existe toutefois pas à notre connaissance d'étude comparative détaillée portant sur la façon dont d'Aulnoy et Carter mettent en œuvre la dimension métapoétique dans leurs textes.

Io. Voir U. Heidmann, "Comparatisme et analyse de discours», p. 102-105. 
récurrent, à savoir celui du Palais de Féerie, dont les représentations changent au fil des textes et les transformations suivent le développement du genre du conte, de son inauguration comme institution littéraire à sa chute, puis à sa rénovation et à sa réhabilitation.

\section{Au seuil du Palais de Féerie: "Gracieuse et Percinet»}

Le Palais de Féerie apparaît pour la première fois chez Marie-Catherine d'Aulnoy dans "Gracieuse et Percinet ». Ce conte relate l'histoire de la princesse Gracieuse, qui, maltraitée par sa marâtre, Grognon, est secourue par le prince Percinet. Celui-ci possède le don de féerie et vit dans un palais du même nom, où il emmène Gracieuse pour la protéger. Elle y découvre une salle aux murs de cristal de roche sur lesquels «son histoire jusqu'à ce jour [...] était gravée " ${ }^{11}$, un exploit attribué à Percinet. Cette salle, et le Palais tout entier, présente une mise en abyme du conte qui désigne de fait Percinet comme son auteur. En effet, le prince amoureux ne se contente pas de mettre en images les événements de la vie de Gracieuse au fur et à mesure qu'ils se produisent; il les connaît par avance. Ainsi, lorsque Gracieuse préfère retourner au palais du Roi plutôt que de demeurer chez Percinet, ce dernier lui annonce ceci: «mon palais sera parmi les morts; vous n'y entrerez qu'après votre enterrement» ${ }^{12}$. Effectivement, Gracieuse ne retournera au Palais de Féerie qu’après avoir été enterrée vivante par Grognon ${ }^{13}$. Percinet conjugue donc le pouvoir des fées sur la vie de Gracieuse, et le pouvoir de l’auteure sur la suite du récit.

Le Palais de Féerie et les événements qui s'y déroulent contiennent ainsi de précieux indices sur la suite du conte. Ils nous renseignent sur les traits constitutifs du genre que l'auteure instaure par l'écriture: importance du thème de l'amour, humour, raffinement, hétérogénéité stylistique, intertextualité avec les auteurs anciens et modernes, et références à des formes artistiques mixtes comme l'opéra. Le Palais de Féerie

II. M.-C. d'Aulnoy, "Gracieuse et Percinet», p. 44.

I2. Ibid., p. 48.

I3. Comme Sylvie Ballestra-Puech le note également dans sa contribution au volume, ce rapprochement entre la féerie et la mort jette une ombre sur la fin du conte. Tout n'est pas rose dans le monde féerique d'Aulnoy; l'autorité et l'indépendance de la femme écrivain ne se conquiert qu'au prix de grands sacrifices. 
présente en effet une parenté avec le palais de Cupidon décrit par La Fontaine en 1669 dans "Les Amours de Psyché et de Cupidon». Les gravures retraçant la vie de Gracieuse rappellent les statues et portraits de Psyché qui décorent ce palais. Cette similitude définit le Palais de Féerie comme un lieu dont la valeur suprême est l'amour. On y donne d'ailleurs un opéra relatant les aventures de Psyché et Cupidon, dans lequel Gracieuse et Percinet sont comparés aux héros de La Fontaine:

L'on vous aime, Gracieuse, \& le dieu d'Amour même

Ne saurait pas aimer au point que l'on vous aime ${ }^{14}$.

Pourtant, la méthode que Percinet recommande à Gracieuse pour voir ce qui se passe au palais de son père consiste bizarrement à "mettre son pied sur le sien $\&$ son petit doigt dans sa bouche ${ }^{15}$. La répétition du possessif obscurcit quelque peu le sens de la phrase mais laisse imaginer une scène assez comique et presque inconvenante (de quelle bouche s'agit-il ?). Le burlesque de la scène laisse à penser que la conteuse s'amuse de ses personnages, et de ses illustres références.

Le Palais de Féerie est donc un espace littéraire, allusif, ludique, qui, à l'instar de l'architecture rococo, s'élabore à partir de matériaux divers, et recourt à la citation et au pastiche ${ }^{16}$. Il reflète la culture des cercles mondains de l'époque, constitués autour de femmes qui en définissent les codes et les références obligées ainsi que les thèmes de prédilection, en particulier celui de l'amour ${ }^{17}$. Les valeurs et l'esthétique illustrées par le Palais de Féerie se retrouveront dans tous les contes de Marie-Catherine d'Aulnoy, où ils seront fréquemment traités de manière subtilement ironique ou "enjouée", comme l'est déjà ici Percinet. De nombreux écrits des conteuses de l'époque reprendront les thèmes et l'esthétique ainsi développés par notre auteure. A l'occasion de la «naissance» du conte

I4. M.-C. d'Aulnoy, «Gracieuse et Percinet», p. 44.

15. Ibid., p. 47.

I6. Jean-Paul Sermain qualifie de "marqueterie» ce principe de composition qui mêle des décors, des genres, des discours et des époques hétérogènes, par lequel le conte «invite le lecteur à découvrir ce qu'il est et ce qu'il fait, son art et ses intentions» (J.-P. Sermain, Métafictions (1670-1730), p. 416).

17. Pour des analyses détaillées du contexte sociohistorique et culturel entourant l'émergence du conte de fées littéraire, voir L. Seifert, Fairy Tales, Sexuality and Gender in France 1690-1715 et P. Hannon, Fabulous Identities. Sur les contes d'Aulnoy en particulier, voir N. Jasmin, Naissance du conte féminin. 
de fées littéraire, d'Aulnoy et ses consœurs dotent le nouveau genre des qualités de réflexivité, intertextualité littéraire, humour et autodérision, et le mettent au service des revendications féminines de l'époque.

En effet, le Palais est un domaine essentiellement féminin où Percinet vit avec sa mère, la Reine, et ses sœurs. De plus, à la fin du conte, lorsque Gracieuse retourne au Palais de Féerie, c'est la Reine qui lui enjoint d'épouser son fils. Et c'est aux genoux de la Reine que se jette la princesse en lui disant «qu'elle pouvait ordonner de sa destinée $\&$ qu'elle lui obéirait en tout " ${ }^{18}$. Après une mort symbolique conforme à la prédiction de Percinet, la renaissance de Gracieuse s'effectue sous l'égide de la Reine. Celle-ci prend à son tour la place de la fée marraine dont les présents détermineront l'avenir de l'héroïne. L'entrée dans le Palais marque ainsi la soumission à l'autorité féminine de la Reine de féerie et non à un père, un époux ou un Roi. Cette dimension subversive sera révélée de manière éclatante dans «La Chatte Blanche». Ce conte présente en effet les mêmes caractéristiques que "Gracieuse et Percinet", mais de manière amplifiée.

\section{Le Palais de Féerie au faîte de sa splendeur: "La Chatte Blanche»}

Le Palais de Chatte Blanche dans le conte du même nom rappelle beaucoup le Palais de Féerie de Percinet:

[...] [s]es murs étaient d'une porcelaine transparente, mêlée de plusieurs couleurs, qui représentaient l'histoire de toutes les fées depuis la création du monde jusqu'alors; les fameuses aventures de Peau d'Ane, de Finette, de l'Oranger, de Gracieuse, de la Belle au bois dormant, de Serpentin Vert $\&$ de cent autres n'y étaient pas oubliées ${ }^{19}$.

Comme le Palais de Féerie, ce château est un espace littéraire qui fonctionne comme miroir du récit et miniature du genre dans lequel il s'inscrit. Chatte Blanche est, comme Percinet avant elle, une figure de l'auteure. Elle aussi connaît à l'avance les événements à venir et guide le prince en conséquence. De plus, son éloquence est fréquemment louée.

I8. M.-C. d'Aulnoy, "Gracieuse et Percinet», p. 55.

19. M.-C. d'Aulnoy, «La Chatte Blanche», p. 165-166. 
Le pouvoir de Chatte Blanche dépasse toutefois largement celui de Percinet. Elle manifeste d'abord sa domination sur le récit avec bien plus de force. En effet, lorsqu'elle redevient femme, le prince se voit réduit au silence alors qu'elle prend la parole pour raconter sa propre histoire à la première personne, sur un nombre de pages à peu près équivalent à celui de la première partie du conte qui relate les aventures du prince. Cette prise de pouvoir linguistique se double d'une prise de pouvoir politique $^{20}$. La princesse refuse la couronne du roi; héritière de six royaumes, elle choisit plutôt d'en donner un au père et à chacun des deux frères du prince, en gardant trois pour elle et son époux. C'est elle qui les gouvernera; la fin du conte nous dit qu'elle «s'y est immortalisée autant par ses bontés $\&$ ses libéralités que par son rare mérite $\&$ sa beauté » ${ }^{21}$, sans aucune mention du rôle du prince. Alors que le Palais de Féerie dans "Gracieuse et Percinet" était un univers à part, totalement distinct du royaume du Roi et de Grognon, ici l'autorité de Chatte Blanche dépasse les frontières de son royaume et prend le dessus sur l'autorité royale et masculine.

Ce triomphe de Chatte Blanche peut être interprété comme un signe du changement de statut du conte littéraire à cette époque par rapport à celle de "Gracieuse et Percinet». Percinet représentait en quelque sorte les prédécesseurs littéraires que d'Aulnoy convoquait pour élaborer son conte. Comme Gracieuse, elle avait besoin d'être ainsi introduite dans le Palais de Féerie. La fin du conte créait toutefois un déplacement significatif; la Reine devenait la nouvelle figure de la conteuse en même temps que la fée marraine de Gracieuse. Ce changement marquait symboliquement l'autonomie du conte et de la conteuse par rapport aux autres genres et auteurs, ce que va confirmer "La Chatte Blanche». Le conte de fées littéraire est toujours un genre nouveau et en formation, mais il est désormais en vogue, comme l'indique le titre du recueil Contes nouveaux ou les fées à la mode. Il dispose de ses propres références, qui sont les contes dont les personnages sont représentés sur les murs du

20. Dans sa contribution au volume, D. Haase souligne l'importance de la parole dans les contes et observe que dans Perceforest, Troylus et Zellandine se racontent leurs histoires respectives. Alors que les personnages principaux sont tous deux des figures auctoriales dans le texte médiéval, le prince de "La Chatte Blanche» est réduit au silence chez Madame d'Aulnoy.

2I. M.-C. d'Aulnoy, «La Chatte Blanche», p. 207. 
palais de Chatte Blanche, à savoir les précédents contes d'Aulnoy comme "Gracieuse et Percinet", mais aussi les contes de Perrault ${ }^{22}$.

Il convient toutefois de nuancer cette célébration du conte et de la conteuse, car elle n'est pas dépourvue d'ironie. Ainsi, Chatte Blanche se présente au prince avec «un miaulis si doux $\&$ si charmant qu'il allait droit au cour " ${ }^{23}$. Contrairement aux animaux doués de parole des fables de La Fontaine et des contes de Perrault, l'éloquence purement féline de l'héroïne de Marie-Catherine d'Aulnoy devient suspecte et presque moqueuse. La tonalité ironique de la description de Chatte Blanche fait écho à celle de la nouvelle dans laquelle le conte est enchâssé, $L e$ Nouveau Gentilhomme Bourgeois. Le conte y est présenté comme l'œuvre de Virginie, une provinciale qui imite les conteuses célèbres sans maîtriser les codes mondains. Replacé dans le cadre plus large de la nouvelle, le triomphe de Chatte Blanche semble donc perdre de son impact dans une perspective féministe. Toutefois, c'est précisément dans ce dispositif énonciatif particulier que réside la force subversive du texte. Car la mise à distance ironique du conte relève d'une stratégie visant à échapper à la censure, comme l'a bien montré Jean Mainil ${ }^{24}$. D’Aulnoy semble se rallier à l'opinion des critiques qui considèrent les contes de fées comme des divertissements futiles et infantiles. Mais c'est justement parce qu'il est marginal et «mineur » que le conte se prête à l'expression des revendications féminines, au contraire des genres établis dont la portée politique est évidente, comme la tragédie ${ }^{25}$. En souscrivant en apparence à une vision condescendante du conte, d'Aulnoy s'offre un espace de liberté, un lieu enchanté qu'elle a construit par l'écriture, où elle peut formuler une critique incisive de la politique maritale et de l'exigence de soumission des femmes à l'autorité masculine. Angela Carter s'inscrit dans la

22. N. Jasmin analyse la présence chez d'Aulnoy d'allusions à sa propre production à côté de références à Perrault ou à La Fontaine, en lien avec le phénomène de mode signalé par le titre. Elle y voit notamment un désir d'affirmer l'indépendance du conte de fées littéraire tout en établissant une parenté avec des auteurs respectés (N. Jasmin, Naissance du conte féminin, p. 191).

23. M.-C. d'Aulnoy, «La Chatte Blanche», p. 169.

24. Jean Mainil montre que l'un des effets de la dynamique à l'œuvre entre «La Chatte Blanche» et la nouvelle enchâssante est l'instauration d'une distance ironique à l'égard du conte (J. Mainil, Madame d'Aulnoy et le rire des fées, p. 185-193).

25. Voir L. Seifert, Fairy Tales, Sexuality and Gender in France 1690-1715, p. 9. 
continuité des stratégies littéraires et de la politique de genre déployées par d'Aulnoy, bien que dans un contexte très différent et à d'autres fins.

\section{La chute du Palais de Féerie: "The Courtship of Mr Lyon" ou le conte en ruines}

Il n'y a pas de Palais de Féerie à proprement parler dans «The Courtship of Mr Lyon". Cependant, la demeure de la Bête peut être considérée comme son équivalent, et elle présente à son tour une mise en abyme délibérée du texte. Son caractère surnaturel et merveilleux transparaît dans "the pervasive atmosphere of a suspension of reality" ${ }^{26}$ constatée par le père de Beauty. Son monstrueux propriétaire se désigne luimême comme "the Beast", nous indiquant que la trame de l'histoire suivra celle de "La Belle et la Bête", d'après le conte de Jeanne-Marie Leprince de Beaumont que Carter a traduit ${ }^{27}$. Toutefois, Beauty trouve dans sa bibliothèque "a collection of courtly and elegant French fairy tales about white cats who were transformed princesses " ${ }^{28}$. Cette référence explicite à la Chatte Blanche nous signale que la maison de la Bête renvoie au Palais de celle-ci, tout autant qu'à la demeure de la Bête de Beaumont. La référence à d'Aulnoy fait bien davantage que de tisser un lien thématique entre les deux textes. Elle signale que Carter use des mêmes procédés que la conteuse, et nous incite à lire entre les lignes du récit pour en percevoir l'ironie. Ainsi lorsque le portail qui sépare la maison du monde extérieur se referme avec un bruit sonore qui fait écho à un autre genre littéraire, celui-ci très anglo-saxon: "that reverberating clang seemed final, emphatic, ominous ${ }^{29}$. Cette phrase, qui pourrait être tirée d'un roman gothique, marque le danger latent de la maison mais l'accumulation emphatique des adjectifs crée un effet de surdétermination parodique et de mélange des genres ${ }^{30}$. La maison est

26. A. Carter, "The Courtship of Mr Lyon», p. 145.

27. Voir M. Hennard Dutheil de la Rochère, Reading, Translating, Rewriting (à paraître).

28. A. Carter, "The Courtship of Mr Lyon», p. 148.

29. Ibid., p. 147.

30. Voir P. Brooke, "Lyons and Tigers and Wolves», p. 71. L'écriture de Carter a été associée par Ch. Bacchilega (Postmodern Fairy Tales) à l'esthétique postmoderne, elle aussi caractérisée par le pastiche et la citation. La juxtaposition d'éléments hétérogènes 
quant à elle décrite comme "a miniature, perfect Palladian house that seemed to hide shyly behind snow-laden skirts of an antique cypress» ${ }^{31}$. Son jardin contient "the faded rag of a white rose» ${ }^{32}$. Le décor hivernal et la rose blanche fanée évoquent un univers figé dans le passé. Ce décor suggère que les références culturelles du conte revisité sont elles aussi figées et stéréotypées. Carter intègre ainsi dans son texte des clichés littéraires issus d'époques et de genres variés, dont elle imite également le style comme les architectes associés au palladianisme s'inspirent des temples romains ${ }^{33}$. La transformation de la Bête marque toutefois un retour brutal à la banalité du monde réel en brisant l'illusion produite par l'artifice de l'écriture. Mr Lyon semble avoir «how strange, a broken nose, such as the noses of retired boxers " ${ }^{34}$. Pour la lectrice qui s'attend à voir apparaître un Prince Charmant, cet époux qui évoque un boxeur à la retraite est bien décevant. Le texte nous incite ainsi à remettre en question nos attentes et idées préconçues par rapport aux genres qu'il mobilise, à commencer par le conte de fées. A cette métamorphose correspond celle de la maison lorsque Beauty y revient après son séjour à Londres:

There was an air of exhaustion, of despair in the house and, worse, a kind of physical disillusion, as if its glamour had been sustained by a cheap conjuring trick and now the conjurer, having failed to pull the crowds, had departed to try his luck elsewhere ${ }^{35}$.

Il n'y a plus de fée régnant sur un Palais de féerie resplendissant, mais seulement un vulgaire illusionniste qui se joue de ses lecteurs. Nous assistons à un véritable désenchantement de la Féerie. Il semblerait que le conte ne soit plus qu'une coquille vide, un genre épuisé, condamné à répéter inlassablement les mêmes clichés. Pourtant, Carter, au moment

rappelle aussi le principe de marqueterie typique du conte de fées tel que le décrit JeanPaul Sermain (Métafictions (1670-1730), p. 413-416). Sur l'hybridité générique du conte comme stratégie de réécriture chez Carter, voir l'article de M. Hennard Dutheil de la Rochère dans ce volume.

31. A. Carter, "The Courtship of Mr Lyon», p. 144.

32. Ibid.

33. Il s'agit d'un élément caractéristique de la poétique de Carter. Si le style raffiné de ce texte correspond bien à la richesse et au classicisme des villas palladiennes, il évoque parfois, on l'a vu, la littérature gothique entre autres. Au contraire, on trouvera des traces du folk tale dans le style plus simple et oralisé de "The Werewolf», par exemple.

34. A. Carter, "The Courtship of Mr Lyon", p. 153.

35. Ibid., p. 152. 
où elle prononce cet arrêt de mort du conte, donne aussi la clé de sa renaissance à travers la transformation de Beauty.

\section{Le Palais de Féerie reconstruit}

A son arrivée dans la maison de la Bête, Beauty s'identifiait à «Miss Lamb, spotless, sacrificial» ${ }^{36}$. L'agneau attendant d'être sacrifié évoque la soumission et la passivité, en lien avec un intertexte biblique très présent dans le récit. Dans les années 70 , on se souvient que de nombreuses critiques récusent les contes de fées sous prétexte qu'ils promeuvent systématiquement de telles qualités chez leurs héroïnes ${ }^{37}$. Mais à Londres, Beauty a changé. Lorsqu'elle observe son reflet dans le miroir, elle voit que « [h] er face was acquiring, instead of beauty, a lacquer of the invincible prettiness that characterises certain pampered, exquisite, expensive cats" ${ }^{38}$. Le lecteur averti réalise alors que l'allusion à Chatte Blanche marque un déplacement de l'attention portée à la métamorphose de la Bête vers celle de Beauty à travers la perception qu'a Beauty d'elle-même. Après s'être identifiée au docile agneau biblique, celle-ci invoque la chatte du conte féminin qui ne se conforme guère aux rôles de genre traditionnels. En effet au début du conte d'Aulnoy, la princesse qui deviendra Chatte Blanche n'a rien d'une créature virginale et obéissante. Sa métamorphose constitue une punition des fées due à son refus d'épouser le fiancé monstrueux qu'elles lui ont choisi. La transformation de Beauty d'agnelle en chatte montre qu'elle possède une semblable capacité à se révolter, un trait de caractère bien éloigné des stéréotypes sur les héroïnes de contes de fées que condamnent les critiques féministes contemporaines de Carter ${ }^{39}$.

Plus que la chute de la maison du conte comme genre désuet et inhabitable pour les auteurs et lecteurs contemporains, c'est donc la vacuité des clichés qui lui sont associés que dénonce la maison de la Bête à l'abandon. Il est aussi illusoire d'imaginer que les contes promeuvent

36. Ibid., p. 148.

37. Voir par exemple M. K. Lieberman, "Some Day My Prince Will Come" ", p. $190-192$.

38. A. Carter, "The Courtship of Mr Lyon», p. 151.

39. Ce potentiel sera pleinement réalisé dans le récit suivant, "The Tiger’s Bride». 
systématiquement la pureté et la passivité féminine que de penser que ces qualités permettront à une femme de transformer une Bête - un homme laid et brutal - en homme séduisant et raffiné. Les stéréotypes de genre associés au conte sont sans doute figés dans des représentations culturelles mais une lecture attentive des textes révèle une complexité et un matériau qu'Angela Carter réutilise dans ses réécritures à l'architecture complexe ${ }^{40}$. Elle en dégage les aspects subversifs et rend ainsi hommage à des femmes auteurs en marge du "canon» masculin (Perrault, Grimm et Andersen), comme Jeanne-Marie de Beaumont à travers «La Belle et la Bête», et Marie-Catherine d'Aulnoy à travers "La Chatte Blanche». En s'inspirant des stratégies déjà mises en place par d'Aulnoy trois cents ans plus tôt et en les adaptant à son propre contexte sociohistorique et culturel, Carter crée de nouveaux effets de sens qui lui permettent à la fois d'articuler un message pertinent pour les lectrices du $\mathrm{XX}^{\mathrm{e}}$ siècle et de souligner les qualités émancipatrices des contes d'Aulnoy. Carter reconstruit donc son propre Palais de Féerie sur les ruines de celui de son prédécesseur. La mort symbolique et la renaissance de Gracieuse, puis la décapitation qui permettait à la Chatte de redevenir femme thématisaient déjà ce double mouvement. Par un jeu subtil sur les références intertextuelles, d'Aulnoy et Carter déconstruisent et recréent simultanément, récupérant les codes des genres littéraires auxquels elles se réfèrent pour mieux les renouveler. Les deux auteures exploitent et renforcent ainsi le don de métamorphose du conte, sa constante création et recréation, et écrivent le destin du genre en même temps que leurs propres textes.

\author{
Magali MonnieR \\ Université de Lausanne
}

40. Jessica Tiffin critique les auteurs féministes, et notamment les détracteurs de Carter qui, en condamnant d'emblée toute tentative de réécriture de contes, perpétuent l'idée erronnée que les contes sont avant tout les vecteurs d'une idéologie patriarcale (J. Tiffin, Marvelous Geometry, p. 70-71). 


\section{BIBLIOGRAPHIE}

\section{Sources}

Aulnoy, Marie-Catherine le Jumel de Barneville, comtesse d', "Gracieuse et Percinet", in Contes de Madame d'Aulnoy, Contes I: Les Contes des Fées, éd. par Ph. Hourcade, Paris, STFM, 1997 [1697], p. 31-56.

—, "La Chatte Blanche», in Contes II: Contes nouveaux ou Les Fées à la Mode, éd. par Philippe Hourcade, Paris, STFM, 1998 [1698], p. 163-208.

Carter, Angela, "The Courtship of Mr Lyon", in The Bloody Chamber and Other Stories, reprinted in Burning Your Boats: Collected Stories, London, Vintage, 2006 [1979], p. 144-153.

\section{Travaux}

Bacchilega, Christine., Postmodern Fairy Tales. Gender and Narrative Strategies, Philadelphia, University of Pennsylvania Press, 1997.

Ballestra-Puech, Sylvie, Les Parques: Essai sur les figures féminines du destin dans la littérature occidentale, Toulouse, Editions universitaires du Sud, 1999.

Benson, Stephen, «Introduction: Fiction and the Contemporaneity of the Fairy Tale", in Contemporary Fiction and the Fairy Tale, ed. by S. Benson, Detroit, Wayne State University Press, 2008, p. 1-19.

-, "Preface to the Special Issue on the Fairy Tale after Angela Carter", Marvels \& Tales: Journal of Fairy-Tale Studies, 24 (2010), p. 13-15.

Böнм, Roswitha, "La participation des fées modernes à la création d'une mémoire féminine», in Les femmes au Grand Siècle. Le Baroque: musique et littérature. Musique et liturgie, éd. par D. Wetsel, F. Canovas, Tübingen, Gunter Narr Verlag, 2003, p. 119-131. 
Brooke, Patricia, "Lyons and Tigers and Wolves - Oh My! Revisionary Fairy Tales in the Work of Angela Carter ", Critical Survey, 16 (2004), p. 67-88.

Carter, Angela, "Notes from the Front Line», in Shaking a Leg, London, Chatto \& Windus, 1997, p. 36-43.

HaAse, Donald, "Feminist Fairy-Tale Scholarship", in Fairy Tales and Feminism: New Approaches, ed. by Donald Haase, Detroit, Wayne State University Press, 2004, p. 1-36.

Hannon, Patricia, Fabulous Identities: Women's Fairy Tales in Seventeenth-Century France, Amsterdam/Atlanta, Rodopi, 1998.

Harries, Elizabeth Wanning, Twice Upon A Time: Women Writers and the History of the Fairy Tale, Princeton, Princeton University Press, 2001.

Heidmann, Ute, "Comparatisme et analyse de discours. La comparaison différentielle comme méthode", in Sciences du texte et analyse de discours. Enjeux d'une interdisciplinarité, éd. par J. Adam, U. Heidmann, Genève, Slatkine, 2005, p. 99-118.

Hennard Dutheil de la Rochère, Martine, " But Marriage Itself is no Party": Angela Carter's Translation of Charles Perrault's "La Belle au bois dormant" " or Pitting the Politics of Experience Against the Sleeping Beauty Myth", Marvels \& Tales: Journal of Fairy Tale Studies, 24 (2010), p. 131-151.

—, Reading, Translating, Rewriting: from Angela Carter's The Fairy Tales of Charles Perrault to The Bloody Chamber, Detroit, Wayne State University Press (à paraître).

Jasmin, Nadine, Naissance du conte féminin. Mots et merveilles: Les Contes de fées de Madame d'Aulnoy (1690-1698), Paris, Honoré Champion, 2002.

Lieberman, Marcia K., " "Some Day My Prince Will Come”: Female Acculturation Through the Fairy Tale", in Don't Bet on the Prince: Contemporary Feminist Fairy Tales in North America and England, ed. by Jack Zipes, Hants, Scolar Press, 1993, p. 185-200.

MaInil, Jean, Madame d'Aulnoy et le rire des fées: essai sur la subversion féerique et le merveilleux comique sous l'Ancien Régime, Paris, Kimé, 2001.

Seifert, Lewis, "Les Fées Modernes: Women, Fairy Tales, and the Literary Field in Late Seventeenth-Century France», in Going Public: Women and Publishing in Early Modern France, ed. 
by E. C. Goldsmith, D. Goodman, Ithaca/London, Cornell University Press, 1995, p. 129-145.

-, Fairy Tales, Sexuality and Gender in France 1690-1715: Nostalgic Utopias, Cambridge, Cambridge University Press, 1996.

Sermain, Jean-Paul, Métafictions (1670-1730). La réflexivité dans la littérature d'imagination, Paris, Honoré Champion, 2002.

Tiffin, Jessica, Marvelous Geometry: Narrative and Metafiction in Modern Fairy Tale, Detroit, Wayne State University Press, 2009.

Verdier, Gabrielle, «Figures de la conteuse dans les contes de fées féminins", XVII e siècle, 180 (1993) p. 481-499.

Warner, Marina, From the Beast to the Blonde: On Fairy Tales and Their Tellers, London, Chatto \& Windus, 1994. 